Gazi University
Pournal of Science
PART C: DESIGN AND TECHNOLOGY
http://dergipark.gov.tr/gujsc

\title{
Developing of a Virtual MCA to Acquire a Radiation Spectrum
}

\author{
Gözde TEKTAŞ1,2,* (1) Cüneyt ÇELIKTAŞ² (10) \\ ${ }^{I}$ Izmir University of Economics Faculty of Arts and Sciences, Department of Physics, 35330, Balçova/IZMIR \\ ${ }^{2}$ Ege University Faculty of Science, Department of Physics, 35100, Bornova/IZMIR
}

\section{Article Info}

Research article

Received:22/02/2021

Revision: $14 / 04 / 2021$

Accepted:20/04/2021

Keywords

Virtual MCA

Real MCA

Pulse Processing

\begin{abstract}
A virtual MCA (Multichannel Analyzer) was developed via a software in the computer environment to display a spectrum for radiation detection experiments. A pulse generator that substitutes for a particle detector was used to supply the signals to be analyzed in the MCA. The pulses from the generator were analyzed by a virtual MCA and a real MCA. Channel numbers and the number of counts in these channels, and the total counts obtained from the virtual MCA and the real MCA were compared with each other. For comparing of the number of counts, data were accumulated in different acquisition times. After comparison of the results, it was observed that the virtual MCA was quite successful as well as a real MCA. It was concluded from the introduced work that the developed virtual MCA would be able to use in a radiation detection system like a real one.
\end{abstract}

\section{INTRODUCTION}

Virtual instrumentation refers to the use of computers and workstations, in combination with data collection hardware devices and virtual instrumentation software, to construct an integrated instrumentation system [1]. In test and measurement areas, virtual instrumentation has been widely adopted [2]. With virtual instruments, engineers and scientists build measurement and automation systems that suit their needs exactly instead of being limited by traditional fixed-function instruments [3].

Nuclear DSP (Digital Signal Processing) systems are commonly realized by the virtual instrumentation technique which is performed in LabVIEW graphical programming environment [4].

LabVIEW is a fully functional graphical programming language which offers a variety of features that simplifies the development of sophisticated applications for control, instrumentation, and data acquisition [5]. Using LabVIEW utilization of virtual instrumentation was possible in electrical engineering applications [6], prototype design in cardiac systolic function [7] and teaching-learning process in engineering [8].

The particles with different energies from a radioactive source generate electric signals with different amplitudes in a radiation detector. However, pulse generators are used to generate the pulses with different amplitudes, shapes, frequencies and so on. For this reason, a pulse generator was used here to stand for the detector signals without any radiation exposure.

Devices which sort out incoming pulses according to pulse heights and keep the number of counts at each height in a multichannel memory are multichannel analyzers (MCA) [9]. They take their input from an analog amplifier and digitize the incoming pulse heights placing the accumulated data into the memory and displaying this pulse height distribution in a histogram [10]. With this specification, they use to acquire the energy spectrum of the incident particles from a radioactive source to a detector. 
In the literature, multichannel pulse height analyzer using field programmable gate array (FPGA) was described by Newton, Ghadigaonkar and D'souza. A graphical user interface was designed using LabVIEW [11]. For nuclear spectroscopy system, a MCA that uses the analog to digital converter (ADC) of a sound card was developed through LabVIEW program [12]. Cao et al. designed a multi-channel and time-division pulse height analyzer based on PXIe bus using 16 bit ADC and FPGA for hard X-ray diagnostic [13]. A FPGA-based digital gamma spectrometer was developed by Liu et al. [14]. For gamma spectroscopy, a systematic literature review of MCA based on FPGA was given by Susanto et al. [15]. An online digital signal processing system based on an FPGA was developed by Kim et al. The system was tested for gamma rays from ${ }^{137} \mathrm{Cs}$ [16]. An environmental gamma spectrometry system was developed by Mitra et al. An in-house designed and developed FPGA based MCA was used [17]. Digital multi-channel analyzer implemented on FPGA was designed by Thuraka, Ganesh and Prakash et al. [18].

In this study, it was aimed to develop a virtual MCA through LabVIEW program in a computer environment. Its specifications (such as count of the pulses) are performed by the software. The data were acquired through a digitizer to the virtual MCA, and a code was written for this data acquisition different from the other referenced works. Pulses acquired from a pulse generator were processed with the virtual MCA and the real MCA. It was investigated whether channel numbers and the number of counts in the virtual MCA were compatible with those of the real one. Thus, the developed virtual MCA was tested whether it would be able to used instead of the real MCA or not.

\section{MATERIALS AND METHODS}

In the present study, a virtual MCA was developed via LabVIEW software in the computer environment in order to use it for a radiation detection experiment to accumulate and analyze the detector signals. In the virtual MCA, the processes such as displaying the spectrum, determination of the number of counts were performed by a written code using the software functions. A part of the block diagram of the virtual MCA is given in Figure 1.

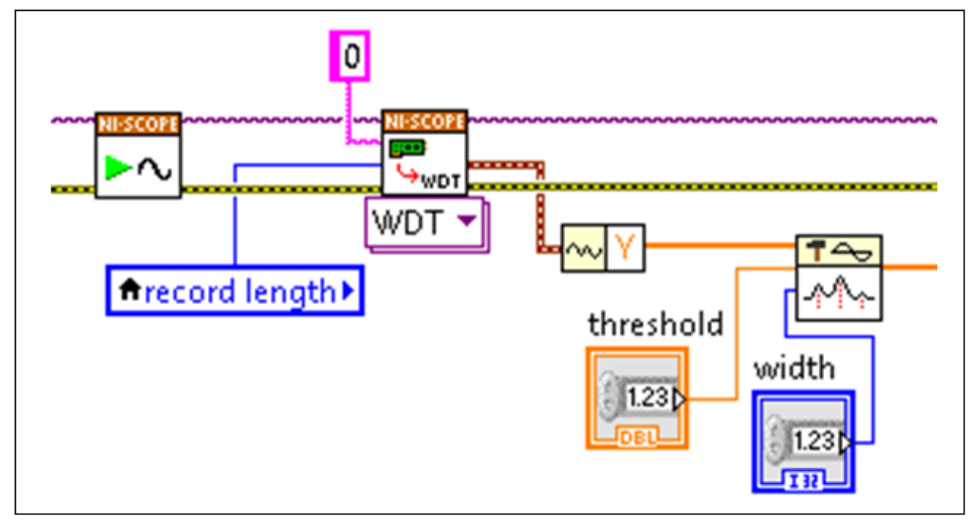

Figure 1. A part of the block diagram of the virtual MCA

The channel numbers and the number of counts in these channels, and the total counts acquired from the virtual MCA were compared with those of a real MCA (ORTEC TRUMP 8k). For this comparison, a pulse generator (ORTEC 419) and an amplifier (ORTEC 485) were used. The pulse generator generates the pulses like a detector output signals. It simulates the detection of a nuclear particle reaction in a semiconductor or scintillation radiation detector, as well as serving as a specialized pulse generator for use with pulse processing instrumentation [19].The amplifier is a general-purpose amplifier that allows operation with semiconductor detectors and scintillation detectors in a wide variety of applications [20]. It has coarse and fine gain switches. The pulses acquired from the generator were sent to the input of the amplifier. After the pulses were amplified by the amplifier, the output pulses were processed by the virtual MCA through a digitizer (NI 5133), and the real MCA. A block diagram used for the measurements can be seen in Figure 2. The pulses with the frequency of $50 \mathrm{~Hz}$ were used in the measurements. 


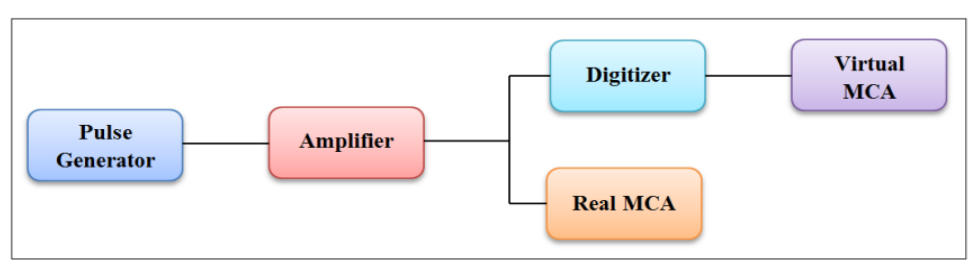

Figure 2. A circuit schema for the measurements

\subsection{Comparison of the Channel Numbers}

To compare the channel numbers of the virtual and real MCAs, the coarse gain value of the amplifier was changed from 2 to 64, and the channel numbers with maximum counts of the accumulated spectra were displayed in both MCAs. The fine gain value of the amplifier was adjusted to 5, which is the mid value, and kept constant, and the acquisition time was $100 \mathrm{~s}$ during this process. Thus, we tested whether the placesof the pulses from the generator, which means channel numbers, were the same in both MCAs.

\subsection{Comparison of the Counts}

The acquisition time was changed to test whether the total counts obtained from both MCAs were compatible with each other. So, it was adjusted to 50, 100, 200 and $300 \mathrm{~s}$. Each measurement was repeated three times to sensitively determine the number of counts for each time value. The counts from both MCAs were accumulated, and their averages were compared with each other.

\section{RESULTS}

For each value of the coarse gain, the channel numbers and the number of counts in these channels are given in Table 1 for both MCAs.

Table 1. Channel numbers and the number of counts in these channels in both MCAs

\begin{tabular}{lllll}
\cline { 2 - 5 } Coarse Gain & Channel Number & Counts & Channel Number & Counts \\
\hline 2 & 15 & 4996 & 15 & 4034 \\
4 & 31 & 4997 & 31 & 2715 \\
8 & 62 & 5000 & 62 & 4193 \\
16 & 84 & 4989 & 83 & 3918 \\
32 & 169 & 4331 & 169 & 3286 \\
64 & 341 & 4031 & 340 & 1827 \\
\hline
\end{tabular}

A certain channel number shifts as the gain value of the amplifier increases in the MCA spectrum. In order to check this shift versus coarse gain values, and to compare the spectrum shapes for the different coarse gain values, obtained spectradisplayed in both MCAs for 8 (min.) and 64 (max.) settings of the coarse gains were given. For these coarse gain values, the displayed spectra are presented in Figures 3 and 4. 


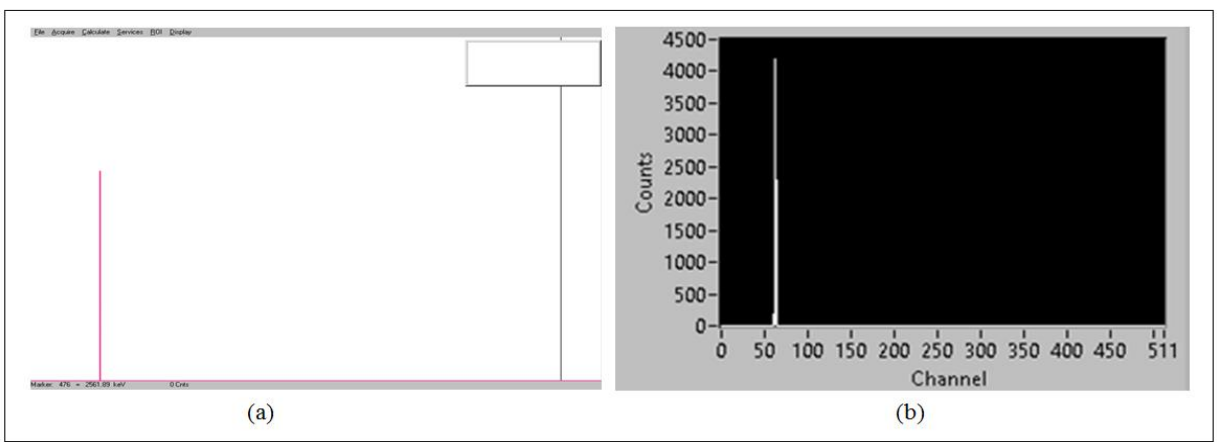

Figure 3. Spectrum shapes for min. (8) setting of the coarse gain in (a) the real MCA and (b) the virtual $M C A$

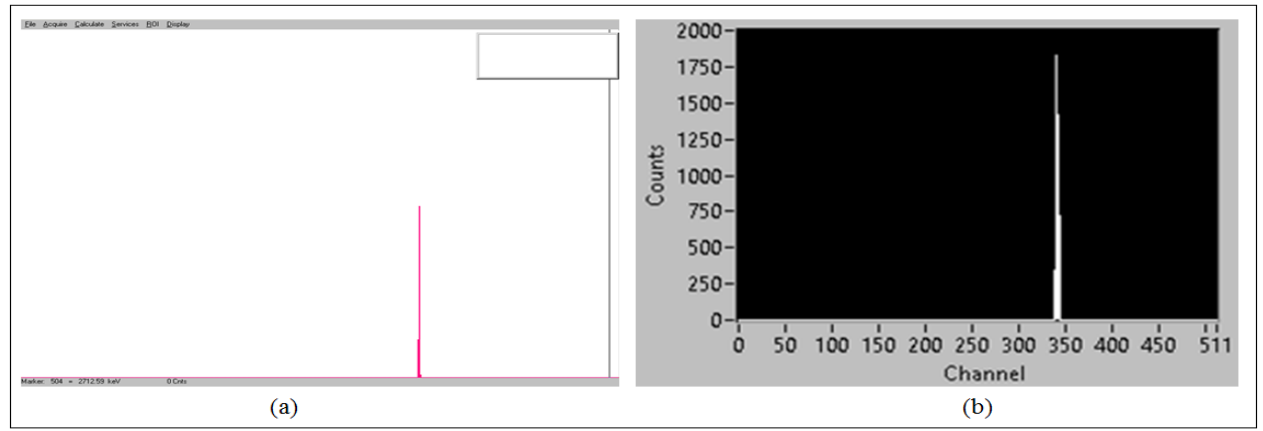

Figure 4. Spectrum shapes for max. (64) setting of the coarse gain in (a) the real MCA and (b) the virtual $M C A$

To test the total count performance of the virtual MCA, the pulses from the generator were accumulated in both MCAs through the amplifier. Accordingly, the averages of recorded total counts from both MCAs are also shown in Table 2 for different acquisition times. The coarse and fine gain values were fixed to 32 and 5 respectively which they are mid ranges of the scales.

Table 2. Average counts obtained from both MCAs for different acquisition times

\begin{tabular}{lll}
\hline Time (s) & $\begin{array}{l}\text { Real MCA } \\
\text { Average Counts }\end{array}$ & $\begin{array}{l}\text { Virtual MCA } \\
\text { Average Counts }\end{array}$ \\
\hline 50 & $2499 \pm 0.577$ & $2501 \pm 0.667$ \\
100 & $4998 \pm 0.000$ & $5001 \pm 0.333$ \\
200 & $9995 \pm 0.882$ & $10001 \pm 0.882$ \\
300 & $14992 \pm 0.882$ & $15001 \pm 0.577$ \\
\hline
\end{tabular}

It was observed that the counts obtained from the virtual MCA increased, as expected, when the acquisition time was increased.

\section{CONCLUSIONS}

In the present study, a virtual MCA was developed via software to be able to use it in a radiation detection experiment. The spectra of a pulse generator were displayed by the virtual and real MCAs. The channel numbers of the spectra versus different gain values, the number of counts acquired for different acquisition times were obtained from both MCAs, and they were compared with each other to test the performance of the virtual MCA.

As can be seen in Table 1, channel numbers and the counts in these channels in response to different gain values from the virtual MCA and the real MCA were quite compatible with each other. When the coarse 
gain value was increased, the spectra in both MCAs were normally shifted to the right, i.e. the higher channel number, because of amplitude increment. While amplitudes of the pulses are determined by the software function in the virtual MCA, this process is performed via the internal electronic circuits in the real MCA. So, the amplitudes of the pulses from the generator were determined in different procedure by both MCAs. For this reason, the pulses were recorded in the different channels in both MCAs, and the counts in these channels were normally not same with each other.

Even though the peak heights, i.e. the number of counts, in the peaks Figures 3 and 4 seem different from each other, they in fact have almost same heights as indicated in Table 1; the difference is due to the appearances of the figures. It can be deduced from the figures that the spectrum shapes displayed in the virtual MCA were rather compatible with the shapes of the real one.

In addition to above, the average counts in different acquisition times acquired from both MCAs were compatible with each other as shown in Table 2. As stated above, the difference of the counts stems from the acquisition procedure of both MCAs.

A while loop was used in the developed code for the virtual MCA.The while loop executes the code it contains until a condition occurs [21]. LabVIEW timer functions use the operating system timers.If the timer function is used to control a loop, differences in the time intervals between each iteration of the loop can be expected depending on the speed of the Central Processing Unit (CPU) of the computer. In addition, several opened windows in the operation system act the while loop speed, affecting the data acquisition performance [22]. For this reason, the number of iteration of the while loop will be different for the same acquisition time. This is the reason why the differences in the total counts were observed in each measurement for the same acquisition time. Furthermore, we could not learn the error sources for the real MCA although we asked the producing company; so, we inferred that its operation procedure details might be confidential. However, as can be seen in Table 2, the errors in the counts obtained from both MCAs were very few.

Consequently, it was deduced from this study that the developed virtual MCA could be used to display the spectrum, to accumulate and record the counts in this spectrum in different acquisition times like a real MCA. Our next work will be on the acquisition the spectrum by the virtual MCA using the detector output signals in a radiation detection setup.

\section{ACKNOWLEDGEMENTS}

This work was supported by Scientific Research Foundation of Ege University under project No. 14 FEN 052.

\section{REFERENCES}

[1] Sumathi, S.,\& Surekha, P. (2007). LabVIEW based advanced instrumentation systems. Berlin: Springer.

[2] Jerome, J. (2010). Virtual instrumentation using LabVIEW. New Delhi: PHI Learning Private Limited.

[3] NI, "Virtual Instrumentation", https://www.ni.com/en-tr/innovations/white-papers/06/virtualinstrumentation.html, Accessed: 17/03/2021.

[4] Folea, S. (2011). LabVIEW-practical applications and solutions: Application of virtual instrumentation in nuclear physics experiments (Jiri Pechousek). Croatia: InTech.

[5] Tooley, M. (2005). PC based instrumentation and control (Third edition). Burlington: Elsevier.

[6] Ramachandiran, G., Kandhasamy, J.,\& Saminathan, A. (2020).Virtual instrumentation in electrical engineering applications. J. Inst. Eng. India Ser. B, 101, 193-199.https://doi.org/10.1007/s40031-02000437-w 
[7] Mâtiu-Iovan, L., Ordodi, V., Ionel, R.,\& Bonciog, D. (2020). Virtual instrumentation based prototype for evaluation of the cardiac systolic function. 2020 International Symposium on Electronics and Telecommunications (ISETC), 1-4.doi:10.1109/ISETC50328.2020.9301047

[8] Aswale, P. S. (2020).Teaching-learning process in engineering using virtual instrument based on LABVIEW. IJETT, 7(1), 15006-15008.

[9] Leo, W. R. (1987). Techniques for nuclear and particle physics experiments. Berlin: Springer-Verlag.

[10] ORTEC, "Pulse-Height, Charge, or Energy Spectroscopy", https://www.ortec-online.com//media/ametekortec/research-electronics-applications/pulse-height-charge-energyspectroscopy.pdf?dmc=1\&la=en\&revision=dc127fad-999f-4b76-b190c5f37964061a\&hash=E49B7ACCA956E39AC647869BC1E4C0F3, Accessed: 17/03/2021.

[11] Newton, F., Ghadigaonkar, V.,\& D'souza, C. (2015).Multichannel pulse height analyser using FPGA for data acquisition. International Journal of Students Research in Technology \& Management, 3(05), 363-364.

[12] Ibrahim, M. M., Yussup, N., Lombigit, L., Rahman, N. A. A.,\& Jaafar, Z.(2014). Development of multichannel analyzer using sound card ADC for nuclear spectroscopy system. AIP Conf. Proc., 1584, 50-53.doi: 10.1063/1.4866103

[13] Cao, H. R., Wang, H. X., Zheng, Y. Y. et al. (2020). Development of digital multi-channel and timedivision pulse height analyzer based on PXIE bus for hard X-ray diagnostic in East. Instrum Exp Tech, 63, 453-460. https://doi.org/10.1134/S002044122004003X

[14] Liu, Y., Xiong, H., Dong, C., Zhao, C., Zhou, Q.,\& Li, S. (2020). Real-time signal processing in field programmable gate array based digital gamma-ray spectrometer. Rev. Sci. Instrum., 91, 104707.https://doi.org/10.1063/5.0005694

[15] Susanto, A. T. et al. (2020). A systematic literature reviews of multichannel analyzer based on FPGA for gamma spectroscopy. J. Phys.: Conf. Ser., 1528, 012016.doi:10.1088/1742-6596/1528/1/012016

[16] Kim, B. J. et al.(2020). Design of optimal digital filter and digital signal processing for a CdZnTe high resolution gamma-ray system. Applied Radiation and Isotopes, 162, 109171. https://doi.org/10.1016/j.apradiso.2020.109171

[17] Mitra, P. et al. (2021). An environmental gamma spectrometry system with CsI(Tl) scintillator and FPGA based MCA for open field deployment. Applied Radiation and Isotopes, 172, 109677. https://doi.org/10.1016/j.apradiso.2021.109677

[18] Thuraka, E. R., Ganesh, R., Prakash, D. B. et al. (2021). Digital multi-channel analyzer for detection and analysis of radiation in nuclear spectroscopy. Materials Today: Proceedings, 38, 3160-3167. https://doi.org/10.1016/j.matpr.2020.09.580

[19] ORTEC, “419 Precision Pulse Generator”, https://www.ortec-online.com/products/electronics/pulsegenerator/419, Accessed: 17/03/2021.

[20] Bryn Mawr College, "Model 485 Amplifier Operating and Service Manual", http://bascom.brynmawr.edu/physics/courses/phys331/resource/manuals/ortec_485_amplifier.pdf, Accessed: 17/03/2021.

[21] NI, "Execution Structures in LabVIEW", https://www.ni.com/getting-started/labviewbasics/execution-structures, Accessed: 17/03/2021.

[22] NI, "Accuracy of Software-Timed Applications in LabVIEW", https://knowledge.ni.com/KnowledgeArticleDetails?id=kA00Z000000P9QiSAK\&l=en-TR, Accessed: 17/03/2021. 